\title{
Protein evolution in deep sea bacteria: an analysis of amino acids substitution rates Stefano Campanaro*†, Laura Treu ${ }^{\dagger}$ and Giorgio Valle
}

\author{
Address: CRIBI Biotechnology Centre, Department of Biology, University of Padua, Via U. Bassi 38/b, 35121, Padua, Italy \\ Email: Stefano Campanaro* - stefano.campanaro@unipd.it; Laura Treu - lauratreu@gmail.com; Giorgio Valle - giorgio.valle@unipd.it \\ * Corresponding author †Equal contributors
}

Published: 13 November 2008

BMC Evolutionary Biology 2008, 8:3/3 doi:10.1/86/I47|-2|48-8-3/3
Received: 3I August 2008

Accepted: 13 November 2008

This article is available from: http://www.biomedcentral.com/|47|-2|48/8/3।3

(C) 2008 Campanaro et al; licensee BioMed Central Ltd.

This is an Open Access article distributed under the terms of the Creative Commons Attribution License (http://creativecommons.org/licenses/by/2.0), which permits unrestricted use, distribution, and reproduction in any medium, provided the original work is properly cited.

\begin{abstract}
Background: Abyssal microorganisms have evolved particular features that enable them to grow in their extreme habitat. Genes belonging to specific functional categories are known to be particularly susceptible to high-pressure; therefore, they should show some evidence of positive selection. To verify this hypothesis we computed the amino acid substitution rates between two deep-sea microorganisms, Photobacterium profundum SS9 and Shewanella benthica KT99, and their respective shallow water relatives.
\end{abstract}

Results: A statistical analysis of all the orthologs, led to the identification of positive selected (PS) genes, which were then used to evaluate adaptation strategies. We were able to establish "Motility" and "Transport" as two classes significantly enriched with PS genes. The prevalence of transporters led us to analyze variable amino acids (PS sites) by mapping them according to their membrane topology, the results showed a higher frequency of substitutions in the extra-cellular compartment. A similar analysis was performed on soluble proteins, mapping the PS sites on the 3D structure, revealing a prevalence of substitutions on the protein surface. Finally, the presence of some flagellar proteins in the Vibrionaceae PS list confirms the importance of bacterial motility as a SS9 specific adaptation strategy.

Conclusion: The approach presented in this paper is suitable for identifying molecular adaptations to particular environmental conditions. The statistical method takes into account differences in the ratio between non-synonymous to synonymous substitutions, thus allowing the detection of the genes that underwent positive selection. We found that positive selection in deep-sea adapted bacteria targets a wide range of functions, for example solute transport, protein translocation, DNA synthesis and motility. From these data clearly emerges an involvement of the transport and metabolism processes in the deep-sea adaptation strategy of both bathytypes considered, whereas the adaptation of other biological processes seems to be specific to either one or the other. An important role is hypothesized for five PS genes belonging to the transport category that had been previously identified as differentially expressed in microarray experiments. Strikingly, structural mapping of PS sites performed independently on membrane and soluble proteins revealed that residues under positive selection tend to occur in specific protein regions. 


\section{Background}

During the last 30 years, a variety of extremophiles have been isolated from abyssal and hadal environments in diverse locations [1]. These habitats are of particular interest since they are characterized by high hydrostatic pressure, low temperature, lack of nutrients and absence of light. Recently, deep sea adaptation has been investigated by sequencing two genomes of psychropiezophilic bacteria which are considered in this study. These cold and pressure-loving microorganisms are Photobacterium profundum strain SS9 [2] and Shewanella benthica strainKT99 [3], hereafter called SS9 and KT99. SS9 belongs to the Vibrionaceae family and is a moderately piezophilic, $\gamma$ proteobacterium, extensively studied for its importance as a model organism for deep-sea adaptation [4]. It was isolated from an amphipod in the Sulu Sea at $2551 \mathrm{~m}$ depth and displays optimum growth at $28 \mathrm{MPa}$ and $15^{\circ} \mathrm{C}$. On the other hand, KT99 is a deep-sea obligate piezophile heterotroph, isolated from a sample taken at $9000 \mathrm{~m}$ depth in the Tonga-Kermadec Trench in the Pacific Ocean [3]. Both bacteria have a remarkable number of phylogenetically closely-related species that are adapted to shallow water conditions. In order to perform a comparative genome analysis we considered only the species for which complete genomes are available, namely $V$. parahaemolyticus, V. fisheri, V. vulnificus for the Vibrionaceae family and $S$. baltica, S. oneidensis, $S$. frigidimarina for the Shewanellaceae family, all of which are mesophilic aquatic bacteria. The Shewanella genus can be subdivided into two major branches, the first one characterized by high-pressure adapted species includes $S$. benthica and the other group characterized by pressure-sensitive species contains $S$. baltica, S. oneidensis and S. frigidimarina [5]. A bioinformatic procedure was applied independently on both families, revealing a valuable number of orthologous genes in each of the two bacterial taxa.

Genetic and biochemical experiments have revealed that both physiological and structural adaptations are essential for high-pressure life. It has been demonstrated that membrane lipids, proteins and solutes accumulation (piezolytes) can influence bacterial growth in deep sea environments [4]. Pressure effects on DNA replication and topology, as well as on cell division, have also been widely discussed as have the nature and regulation of genes that are important for pressure-sensing and the relevance of the transport process in piezophilic bacteria [6].

The object of this study is to further characterize the effect of abyssal conditions on the evolution of SS9 and KT99 genomes. It is not obvious to establish the biological, physical and chemical parameters that are relevant to protein adaptation because abyssal and shallow water environments are extremely different in term of pressure, temperature, light and nutrients. This is an important point to be considered when the two abyssal species described in this paper are compared to phylogenetically related mesophilic species for which our knowledge on their optimal growth conditions is not always fully established. In fact, we are not able to exclude that other parameters, different from hydrostratic pressure, play a role in protein adaptation. Temperature, for example, could influence our analysis as it is one of the parameters that separates $P$. profundum SS9 from the other shallowwater bacteria considered in our comparison. This parameter has probably less influence in shewanellaceae comparison since growth curves determined for shewanellaceae [5] indicate that only $S$. oneidensis can be considered a mesophilic bacterium, whereas $S$. benthica, $S$. frigidimarina and $S$. baltica are psychrophiles. Furthermore all shallow-water bacteria considered in our comparison contain the deoxyribodipyrimidine photo-lyase gene involved in repair of UV radiation-induced DNA damage, whereas the SS9 and KT99 genomes do not code for this enzyme, which is consistent, since there is a distinct absence of sunlight in their normal deep sea environment.

In Table 1 are summarized the main features of the bacteria considered in our study. Growth optimum was obtained from [7] for P. profundum SS9, from [8] for $V$. parahaemolyticus, from [5] for S. baltica, S. benthica e S. frigidimarina, while growth data for $S$. oneidensis were kindly provided by Daniel I. of the University Claude Bernard (Lyon).

We investigated the presence of positively selected genes in the two bacteria adapted to an abyssal environment, using as a control the phylogenetically related mesophilic species. The computation most frequently used to carry out this analysis is the non-synonymous to synonymous substitution rate ratio, defined omega $(\omega)[9,10]$. In general, an excess of non-synonymous substitutions (dN) over synonymous substitutions (dS) is considered a clear indicator of positive natural selection, because non-synonymous mutations are typically subject to strong purifying selection, whereas synonymous changes are typically neutral.

It should be considered that if we take any two species we would expect to find some genes that underwent positive selection due to the respective evolutionary history of each species. In this study we consider two families of bacteria, each represented by four species. We can assume that some genes underwent positive selection during the evolution of each species, whereas other genes underwent positive selection in clades including more than one species. To identify the genes that underwent positive selection during the evolution of a species we designed the following approach. Firstly, we chose the species for which we wanted to detect genes that underwent positive 
Table I: Features of the bacteria considered in this paper

\begin{tabular}{|c|c|c|c|c|c|c|}
\hline Strain & RefSeq acession no. & Source & $\mathbf{T}_{\text {opt }}$ & Photolyase & $\mathbf{P}_{\text {opt }}$ & Orthologs \\
\hline Photobacterium profundum SS9 & NC_006370 & Sulu Sea & $15^{\circ} \mathrm{C}$ & A & $28 \mathrm{MPa}$ & Vs \\
\hline Vibrio fischeri ESII4 & NC_006840 & Squid symbiont & $30^{\circ} \mathrm{C}$ & $P$ & ND & 3028 \\
\hline Vibrio parahaemolyticus RIMD & NC_004603 & Osaka & $20-43^{\circ} \mathrm{C}$ & $P$ & $0.1 \mathrm{MPa}$ & 3469 \\
\hline Vibrio vulnificus Yj0I6 & NC_005139 & Taiwan & $30-40^{\circ} \mathrm{C}$ & $P$ & ND & 3362 \\
\hline Shewanella benthica KT99 & - & Tonga-Kermadec Trench & $4-15^{\circ} \mathrm{C}$ & $A$ & $50 \mathrm{MPa}$ & Vs \\
\hline Shewanella baltica OSI55 & NC_009052 & Baltic Sea & $4^{\circ} \mathrm{C}$ & $P$ & $10 \mathrm{MPa}$ & 3167 \\
\hline Shewanella oneidensis MR-I & NC_004347 & Oneida lake & $30^{\circ} \mathrm{C}$ & $P$ & $0.1 \mathrm{MPa}$ & 2651 \\
\hline Shewanella frigidimarina NCIMB 400 & NC_008345 & North Sea & $20-22^{\circ} \mathrm{C}$ & $P$ & $0.1 \mathrm{MPa}$ & 2909 \\
\hline
\end{tabular}

Columns report respectively: strain, $N C B$ accession number, isolation site, optimum growth temperature $\left(T_{\text {opt }}\right)$, presence $(P)$ or absence $(A)$ of the deoxyribodipyrimidine photo-lyase gene, optimum growth pressure (ND = Not Determined) and number of orthologous genes identified for each comparison. The omission of the ORFs encoding light-activated photolyase genes from the SS9 and KT99 genomes is due to the absence of sunlight in the deep sea.

selection, in our case the deep-sea bacteria. Secondly, we compared the chosen species with the other three control species of the same family, calculating the $\omega$ values for each orthologous pair. Thirdly, we performed the calculation of the $\omega$ values also within the three control species. Finally, we selected the genes that resulted positive (i.e. underwent positive selection) in each of the three comparisons of the chosen species versus the control species but were negative in the three comparisons within the control species.

It is important to consider that it is likely that the genes selected by this approach specifically underwent positive selection in the chosen species, but this does not automatically imply that they are involved in deep-sea adaptation. This point should be considered for a correct interpretation of the results.

It is known from the literature that evolution of bacterial genes is influenced by several factors, such as protein expression level, functional class and metabolic cost of amino acid residues [11]. Therefore we further considered the distribution of Codon Adaptation Index (CAI) versus $\omega$ values for each orthologous gene, both in Shewanellaceae and Vibrionaceae. It had been previously defined that CAI is negatively correlated with $\mathrm{dN}$ [11]. In fact, proteins expressed at high levels are generally associated with the usage of the "best" synonymous codons, resulting in a direct influence of the codon usage bias on the rate of non-synonymous substitutions in bacteria.

Genome-wide studies have already identified a number of biological processes involved in high pressure adaptation [12]. We used genes that we obtained from the comparison between piezophiles and mesophiles (PS genes) to establish which Gene Ontology (GO) and Cluster of Ortologous Groups (COG) functional classes are targeted by natural selection in bacterial evolution. Moreover PS proteins, belonging to particular categories, can easily show PS sites mainly localized in specific domains. In order to better define the role of amino acid substitutions on deep sea adaptation, we mapped PS sites on the 3D structure as well as on protein topology in respect of transmembrane regions.

\section{Results}

In order to identify genes under positive selection in deepsea adapted bacteria we analyzed 4 Vibrionaceae and 4 Shewanellaceae from which we selected 2,174 orthologous genes shared by the former and 2,180 shared by the latter. These numbers are high enough to allow us to explore all the main biochemical and physiological cellular processes. The two groups were obtained by firstly considering the three mesophilic bacteria $V$. parahaemolyticus, $V$. fisheri and V. vulnificus for the comparison with P. profundum and secondly by using $S$. baltica, $S$. oneidensis and $S$. frigidimarina for the comparison with $S$. benthica (see details in Table 1).

For each orthologous gene pair we aligned DNA sequences and computed amino acid substitution rate. Most of $\omega$ values were included in the 0 to 0.2 range (Figure $1 \mathrm{~A}$ ) and the median $\mathrm{dN} / \mathrm{dS}$ calculated was 0.08 in Vibrionaceae and 0.07 in Shewanellaceae. Using Spearman statistics we verified the inverse correlation between CAI and $\omega$ values achieving $\rho$ coefficients included in the -0.27 to -0.33 range in Vibrionaceae ortholog comparison and -0.18 to -0.36 in Shewanellaceae (Figure 1B). Moreover PS genes do not have a biased CAI value and their distribution is in accordance with those of all the orthologs. This homogeneous distribution confirms that the $\omega$ values of PS genes are not determined by low CAI values but mainly by deep sea adaptation.

It is known that orthologs generally have low $\omega$ values $(<$ $0.05)$, which implies that the proteins are subject to rela- 

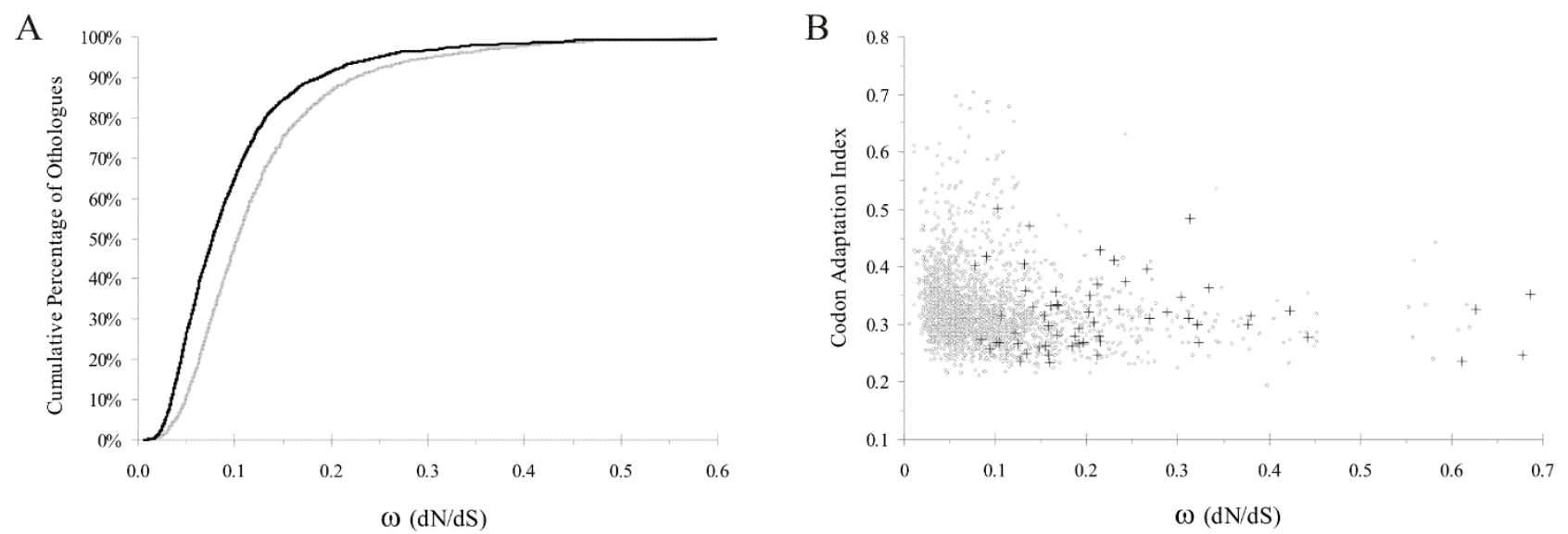

\section{Figure I}

(A) Cumulative codon rate ratios; (B) distribution of $\omega$ values in comparison with CAl. A) The figure shows cumulative (percentage) codon rate ratios for total Shewanellaceae and Vibrionaceae orthologs. S. benthica vs. S. frigidimarina and $P$. profundum vs. V. vulnificus cumulative $\omega$ ratios are represented respectively by black and gray lines. B) Distribution of the $\omega$ (dN/ $\mathrm{dS}$ ) values computed between the orthologous genes of $S$. benthica and S. frigidimarina in comparison with the Codon Adaptation Index calculated for S. benthica. The Spearman correlation coefficient $(\rho)$ calculated between $\omega$ and CAI values is -0.32 . PS genes identified in KT99 are highlighted in black. The graph reported is representative of those calculated in all other organisms.

tively strong purifying selection. Genes with $\mathrm{dN} / \mathrm{dS}>1$ are formally defined as being subject to positive selection, in fact amino acid changes are accumulating faster than would be expected given the underlying silent substitution rate. Even so, proteins with $\mathrm{dN} / \mathrm{dS}<1$ may still contain sites under positive selection, but their contribution to the $\mathrm{dN} / \mathrm{dS}$ for the whole protein is masked by the purifying selection at other sites [13]. To overcome this problem we only considered the orthologous genes having values significantly higher in the comparison between piezophilic and mesophilic bacteria, with respect to the comparison within mesophiles. Thus, we identified 213 PS genes in the Vibrionaceae family and 61 in Shewanellaceae respectively (see report in Additional files 1, 2). The list includes many different functional classes of proteins, ranging from transporters, that are definitively the most represented, to metabolic enzymes, chaperons and ribosomal proteins.

\section{Processes Involved in Deep Sea Adaptation}

To better understand the role of these genes in the evolution of extremophiles all orthologs were assigned to functional categories according to COG and GO annotations $[14,15]$. The evidence of PS genes enrichment in COG specific groups was calculated using hypergeometric distribution. Furthermore we used the Fisher exact test on GO in order to give an overview of bacterial adaptation at a higher level of detail, for example specific biological mechanisms. In fact COGs give a more general idea of the processes involved. We obtained two COGs showing evidence of positive selection both in SS9 and KT99: "Nucleotide transport and metabolism" (F) and "Inorganic ion transport and metabolism" (P). There are three additional COG categories only enriched in SS9: "Cell wall/membrane/envelope biogenesis" (M), "Intracellular trafficking, secretion and vesicular transport" (U), "General function prediction only" (R). The "Defense mechanisms" (V) class is KT99 specific, as shown in Table 2. From these data clearly emerges an involvement of the transport and metabolism processes in the deep-sea adaptation strategy of both piezophiles considered in this study, while the adaptation of other biological processes seems to be peculiar to either one or the other.

A similar analysis was performed using the Gene Ontology classification (GO) that takes into account biological processes, cellular components and molecular functions. Both bacterial families reveal an enrichment of PS genes belonging to the "Localization" process, that is the action by which a substance or other structures are transported to (or maintained in) a specific location, see details in Additional file 3. In GO this term is strictly related to "Transport" activity. It is relevant that there are specific "Transport processes" enriched in both piezophiles, instead "Protein folding" and "Cell motility" are present only in SS9. Several genes belonging to the last category are involved in the flagellar basal structure as shown in KEGG representation on Additional file 4[16]. The only 
Table 2: COG categories enriched with PS genes in KT99 and SS9

\begin{tabular}{|c|c|c|c|c|c|c|c|}
\hline Functional Categories & COGs & KT99 & tot & p-value & SS9 & tot & p-value \\
\hline Energy production and conversion & $\mathrm{C}$ & 5 & 149 & 0.214 & 12 & 125 & 0.482 \\
\hline Cell cycle control, cell division, chromosome partitioning & $\mathrm{D}$ & I & 28 & 0.175 & 2 & 23 & 0.407 \\
\hline Amino acid transport and metabolism & $\mathrm{E}$ & 7 & 173 & 0.093 & 22 & 200 & 0.261 \\
\hline Nucleotide transport and metabolism & $\boldsymbol{F}$ & 4 & 48 & 0.009 & 10 & 60 & 0.032 \\
\hline Carbohydrate transport and metabolism & G & I & 50 & 0.396 & 9 & 91 & 0.424 \\
\hline Coenzyme transport and metabolism & $\mathrm{H}$ & 2 & 114 & 0.606 & 4 & 107 & 0.987 \\
\hline Lipid transport and metabolism & I & 0 & 82 & - & 6 & 65 & 0.476 \\
\hline Translation, ribosomal structure and biogenesis & J & 5 & 124 & 0.117 & 9 & 125 & 0.817 \\
\hline Transcription & $\mathrm{K}$ & 0 & 126 & - & 10 & 136 & 0.816 \\
\hline Replication, recombination and repair & L & 3 & 107 & 0.330 & 5 & 100 & 0.945 \\
\hline Cell wall/membrane/envelope biogenesis & M & 4 & 106 & 0.158 & 16 & 113 & 0.050 \\
\hline Cell motility & $\mathrm{N}$ & I & 73 & 0.596 & 9 & 75 & 0.210 \\
\hline Posttranslational modification, protein turnover, chaperones & $\mathrm{O}$ & 2 & 111 & 0.587 & 10 & 102 & 0.441 \\
\hline Inorganic ion transport and metabolism & $\boldsymbol{P}$ & 4 & 72 & 0.044 & 16 & 107 & 0.033 \\
\hline Secondary metabolites biosynthesis, transport and catabolism & $\mathrm{Q}$ & 0 & 34 & - & I & 28 & 0.786 \\
\hline General function prediction only & $\mathbf{R}$ & 4 & 209 & 0.683 & 29 & 217 & 0.035 \\
\hline Function unknown & $S$ & 19 & 659 & 0.319 & 52 & 577 & 0.789 \\
\hline Signal transduction mechanisms & $\mathrm{T}$ & 0 & 105 & - & 11 & 116 & 0.494 \\
\hline Intracellular trafficking, secretion, and vesicular transport & $\mathbf{U}$ & 2 & 74 & 0.325 & 13 & 81 & 0.027 \\
\hline Defense mechanisms & $\mathbf{v}$ & 3 & 25 & 0.004 & 2 & 36 & 0.713 \\
\hline Total Orthologs & & 67 & 2469 & & 213 & 2484 & \\
\hline
\end{tabular}

Columns represent respectively: functional classes, COG codes, number of PS genes for each category, total number of orthologous genes for each category and p-value calculated using hypergeometric distribution for KT99 (columns 3, 4, 5) and SS9 (columns 6, 7, 8). In bold are highlighted significant values $(p=0.05)$ and the italics indicate classes enriched in both bacteria.

significant Cellular Component obtained from the analysis in Shewanellaceae was the "Membrane" category. It emerges also that in Shewanellaceae there is a higher number of enriched categories, but most of them contain only a single gene. For this reason we considered them less noteworthy, even if we cannot exclude that their specific role in the process of adaptation may have been relevant. All genomic analyses were done separately in both bacterial families. This allowed the identification of individual adaptation mechanisms that developed independently in the two extremophiles considered as well as those in common. In fact examining the two lists of PS genes we found only 12 shared by both families, corresponding respectively to $5.6 \%(12 / 213)$ in SS9 and to $18 \%(12 / 61)$ in KT99, see details in Table 3 . As a validation of the previous results these genes belong to the most relevant classes: Transport, Membrane and Cell motility. These proteins will be further considered in the Discussion section, due to their highly relevant role in adaptation to these extreme environmental conditions.

\section{Localization of PS Sites on Protein Structure}

For a better comprehension of the variable amino acids functional role we mapped them on protein structures, domains and trans-membrane regions. We identified amino acids specifically different from a chemical-physical point of view in piezophiles, compared to mesophiles. Hereafter we will call them PS sites.
Position analyses were done using two distinct strategies for soluble and membrane proteins, due to the different meaning of amino acid substitutions in these categories. Among the 213 PS proteins identified in Vibrionaceae family, 65 of them have predicted trans-membrane regions and 149 are predicted as soluble. By querying the ModBase database [17] we obtained 42 models having more than $40 \%$ similarity with known protein structures. Using these models we mapped the PS sites on the $3 \mathrm{D}$ structure of soluble proteins using PyMol software (Figure 2).

Generally the variable amino acids are localized on the protein surface. To confirm their distribution, we evaluated the number of solvent exposed PS sites (Additional file 1). This computation was performed for all SS9 models with the exception of PBPRA0158, as it is trans-membrane.

In 27 proteins more than $75 \%$ of the PS sites are located on the surface while in other 14 cases they fall in the $50 \%$ - $75 \%$ range. We verified that the variable amino acids are predominantly solvent exposed, as reported in Figure 3. Moreover, considering the total amino acid number, we found 4 proteins with more than $75 \%$ of solvent exposed residues, $37 \mathrm{fell}$ in the $50 \%-75 \%$ range and one had less than $50 \%$ exposed residues. All these results indicate that in only three cases the fraction of solvent exposed PS sites 
Table 3: Common orthologous genes identified as positive selected and shared by KT99 and SS9

\begin{tabular}{|c|c|c|c|c|}
\hline $\mathbf{N}^{\circ}$ & KT99 Function & Locus Tag & COG & $q(\%)$ \\
\hline $\mathbf{I}$ & Molybdenum ABC Transporter, Permease Protein & KT99_02056 & $4 \mid 49 P$ & 10.0 \\
\hline 2 & Hypothetical Protein & KT99_20194 & - & 0.0 \\
\hline 3 & Phosphoribosylaminoimidazole Carboxylase, Catalytic Subunit & KT99_I702I & $004 \mathrm{IF}$ & 0.0 \\
\hline 4 & Dithiobiotin Synthetase & KT99_10643 & $0132 \mathrm{H}$ & 4.9 \\
\hline 5 & Uracil Permease & KT99_16519 & $2233 \mathrm{~F}$ & 0.0 \\
\hline 6 & TonB2 Protein & KT99_09573 & $0810 \mathrm{M}$ & 10.0 \\
\hline 7 & Primosomal Replication Protein N, Putative & KT99_007I0 & - & 7.0 \\
\hline 8 & Methylated DNA-Protein Cysteine Methyltransferase & KT99_I5I70 & 0350L & 7.0 \\
\hline 9 & NADH Dehydrogenase & KT99_I3367 & $1252 \mathrm{C}$ & 0.0 \\
\hline 10 & Succinate-Semialdehyde Dehydrogenase & KT99_19874 & $1012 \mathrm{C}$ & 0.0 \\
\hline II & MSHA Biogenesis Protein MshL & KT99_07703 & $1450 \mathrm{NU}$ & 10.0 \\
\hline 12 & RND Multidrug Efflux Transporter MexF & KT99_04334 & 084IV & 10.0 \\
\hline $\mathbf{N}^{\circ}$ & SS9 Function & Locus Tag & COG & $q(\%)$ \\
\hline $\mathbf{I}$ & Putative Phosphate ABC Transporter, Permease Protein & PBPRAI 392 & $058 \mathrm{IP}$ & 4.3 \\
\hline 2 & Hypothetical Protein & PBPRA2020 & - & 4.8 \\
\hline 3 & Hypothetical Phosphoribosylaminoimidazole Carboxylase, Catalytic Subunit & PBPRA3574 & $004 \mathrm{IF}$ & 0.0 \\
\hline 4 & Dithiobiotin Synthetase & PBPRA2326 & $0132 \mathrm{H}$ & 8.3 \\
\hline 5 & Putative Xanthine/Uracil Permease & PBPRA0I86 & $2233 \mathrm{~F}$ & 8.3 \\
\hline 6 & Hypothetical TonB Protein & PBPRA2 103 & $0810 \mathrm{M}$ & 4.4 \\
\hline 7 & Hypothetical Primosomal Replication Protein N & PBPRAIOIO & - & 8.3 \\
\hline 8 & Hypothetical Methylated DNA-Protein Cysteine Methyltransferase & PBPRB02I0 & 0350L & 4.3 \\
\hline 9 & Putative Nitrite Reductase $(\mathrm{NAD}(\mathrm{P}) \mathrm{H})$, Large Subunit & PBPRAI 428 & I25IC & 8.3 \\
\hline 10 & Putative Succinylglutamate 5-Semialdehyde Dehydrogenase & PBPRA029I & $1012 \mathrm{C}$ & 3.3 \\
\hline I I & Hypothetical Flp Pilus Assembly Protein & PBPRA2496 & $4964 U$ & 3.3 \\
\hline 12 & Putative Multidrug Resistance Protein & PBPRA272I & $084 I V$ & 4.3 \\
\hline
\end{tabular}

In the first column orthologs are ordered correspondingly in the two families. The NCBI annotation, locus tag, COG categories and SAM significance $q$-value are reported in columns $2-5$. q-value is obtained from SAM software and it is the lowest False Discovery Rate at which the gene is called significant.

is lower or equal to the one calculated for the entire structure. Finally, in nine cases we detected PS sites involving ligand binding sites.

In the analysis of the PS sites of membrane proteins, particular relevance was given to their position. We looked at protein topology, inside or outside the membrane, and at trans-membrane helices. We found 21 proteins with a higher number of PS sites in trans-membrane region, 18 in the external region and 22 in the cytoplasmic region. However these numbers are heavily dependent on the correspondent region length and normalization was required to obtain comparable results between different proteins. The outcome indicates that the higher fraction of PS sites is externally located in $41 \%$ of proteins, cytoplasmic in $35 \%$ and trans-membrane in 24\% (Figure 4A). Moreover, comparing membrane PS sites with extracellular and cytoplasmic together, only $33 \%$ of the proteins had the higher variable amino acids in the trans-membrane helices. Finally, we calculated the mean number of cytoplasmic, external and trans-membrane PS sites normalized with respect to each portion length obtaining respectively 1.03 , 1.06 and 0.86 . Due to their relevance in our study, we also executed the same procedure considering only transporters, in this way we got higher mean values, respectively $1.02,1.28$ and 0.77 for cytoplasmic, external and transmembrane regions. Among the PS genes of Shewanellaceae we could only analyze 12 trans-membrane proteins because no 3D model of soluble proteins was available in ModBase. Despite the low number of genes, structural mapping of PS sites on trans-membrane proteins revealed that the residues under positive selection occur preferentially in the extracellular region (5 proteins), as shown in Figure 4B. Calculating the mean number of normalized cytoplasmic, external and trans-membrane PS sites, we obtained respectively $1.05,0.99$ and 0.91 .

It has to be considered that only some portions of the proteins are under positive selection and this influences the $\omega$ value computed for the entire sequence. We searched for genes under intensive selective pressure to recognize regions with overrepresented nonsynonymous mutations unevenly distributed along the alignment, previously unidentified using conventional rate comparison analysis [18]. We were interested in genes with overabundant mutation regions localized in specific domains that were 

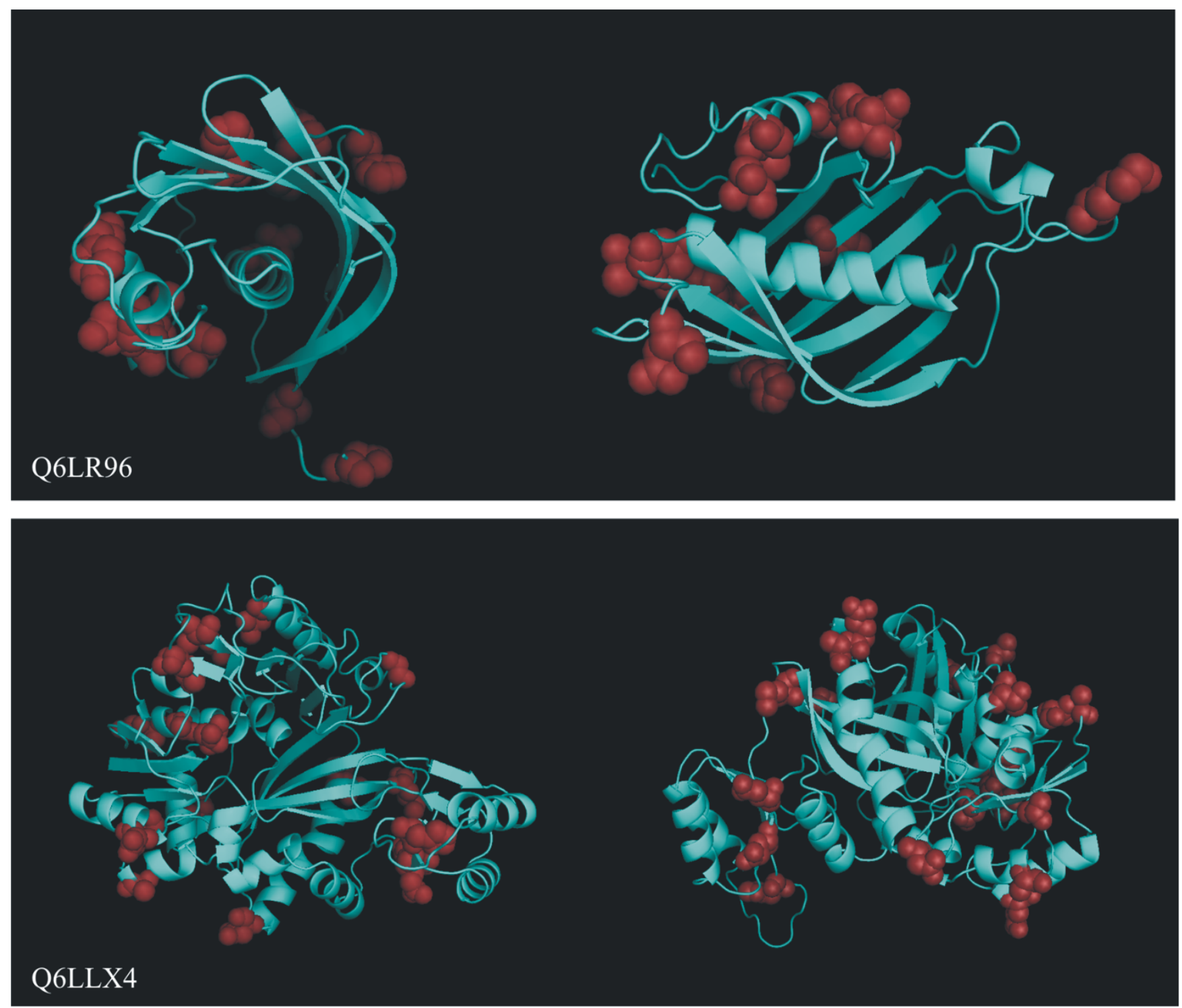

\section{Figure 2}

Distribution of PS sites on two protein structures. Modeled structure of Q6LR96 protein (3-hydroxydecanoyl-ACP dehydratase) and of Q6LLX4 (phosphoribosylamine-glycine ligase) coded respectively by P. profundum SS9 PBPRAI773 and PBPRA3420 genes. Sites showing evidence of positive selection $(P<0.0 \mathrm{I})$ are depicted as red spheres. From two different perspectives it appears that most of PS sites are located on the protein surface.

present in all the comparisons between piezophiles and mesophiles, but absent in mesophilic bacteria only. We discovered just one fast evolving region (FER) in PBPRA0616 among all Vibrionaceae orthologs and we found 10 genes with overabundant mutation rate (OMR). Four of these are trans-membrane proteins and six are soluble proteins. In KT99 we found one FER in the KT99_15170 gene, whilst one trans-membrane protein and five soluble proteins have OMRs (Additional files 1 and 2).

Finally we investigated if the SS9 PS proteins had been previously identified as involved in high pressure and low temperature adaptation and we compared our data with SS9 microarray experiments results [6] and with genes found by systematic mutagenesis screening [19]. Among the 14 PS genes found differentially expressed by microarray analyses, 4 of them were identified as up-regulated under high hydrostatic pressure $(28 \mathrm{MPa})$ compared to 


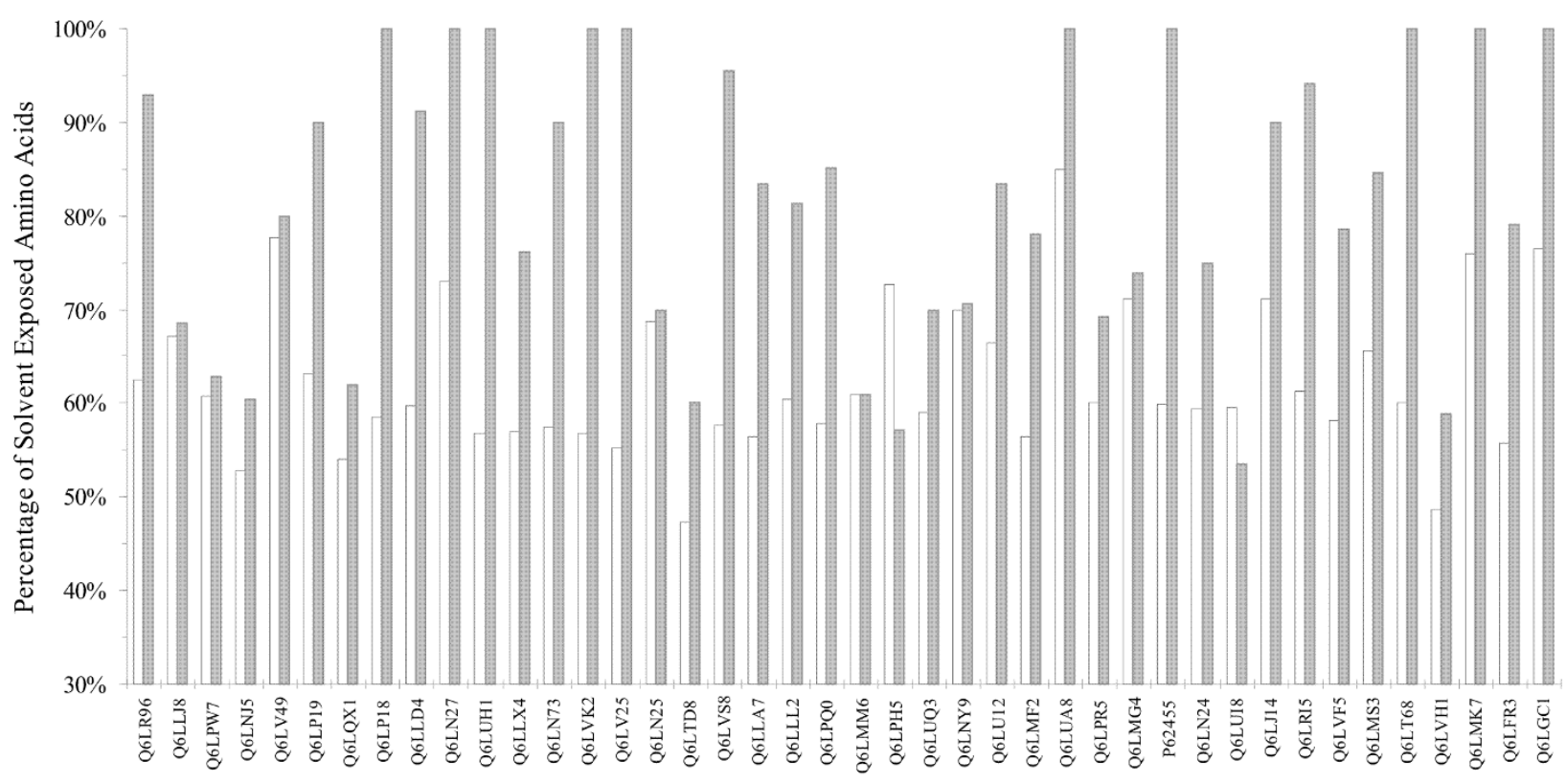

Figure 3

Percentage of solvent exposed PS sites for soluble proteins. In this histogram are reported the percentages of solvent exposed amino acids (white columns) and those of solvent exposed PS sites (grey) for each modeled protein. All values were calculated for SS9 proteins.

atmospheric pressure $(0.1 \mathrm{MPa})$ and 7 were found to be down-regulated; 2 were down-regulated at $4{ }^{\circ} \mathrm{C}$ compared to $16^{\circ} \mathrm{C}$. Two genes, PBPRA2089 and PBPRA0281, were identified in the list from the systematic mutagenesis screening as responsible for piezosensitivity in SS9 mutants.

\section{Discussion}

Vibrionaceae and Shewanellaceae represent a significant portion of the culturable heterotrophic bacteria of oceans, coastal waters and lakes. Some species belonging to these families also inhabit deep-sea environments and, due to the increasing interest in high pressure adaptation, various studies have been undertaken to clarify their characteristics from a genomic point of view [12]. Our study is based on an exhaustive comparison of orthologous genes. It is therefore important to discuss the criteria used for their selection. Two main factors affect the number of orthologous genes shared amongst the considered species: the number of species and their phylogenetic distance. Therefore, we kept the two bacterial families separated and selected two independent sets of orthologous genes, one for each of the two families. Furthermore, for each family we limited the number of species to four: one from deep sea and three from shallow water. In both families we obtained about 2,180 shared orthologous genes. The general rationale of our study is to identify, independently for each family, the genes that underwent positive selection in the deep-sea adapted species. Thus, using Gene Ontology associations, we should be able to see whether any biological processes or molecular functions are particularly affected in one or both bacterial families.

\section{PS Genes Detection}

In both families about 2180 ortologues were identified and utilized in six different two by two $\omega$ value calculations, three between piezophiles-mesophiles and three between mesophiles, the latter considered as a negative control. The level of divergence among the species of a bacterial family is high enough to detect the signature of selection, yet low enough to have a high number of orthologous genes. In previous works computations performed using the $\mathrm{dN} / \mathrm{dS}$ substitution rate were complicated by the uncertainty of the threshold to be considered for genes under positive selection. For this reason, instead of a direct selection of all the instances above a given threshold, we decided to consider only those genes that had statistically higher $\omega$ values in the three piezophilemesophiles comparisons. The approach employed 


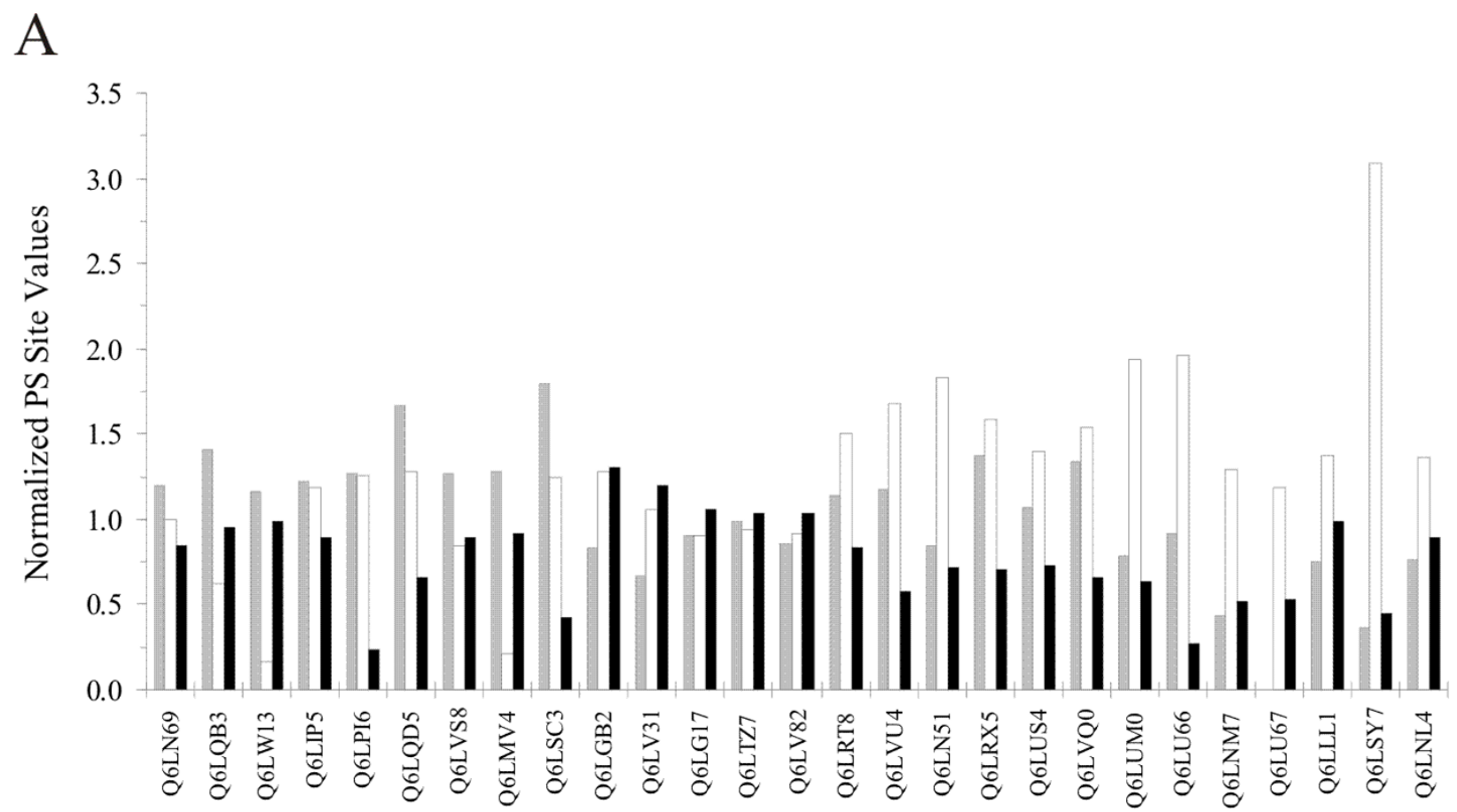

B

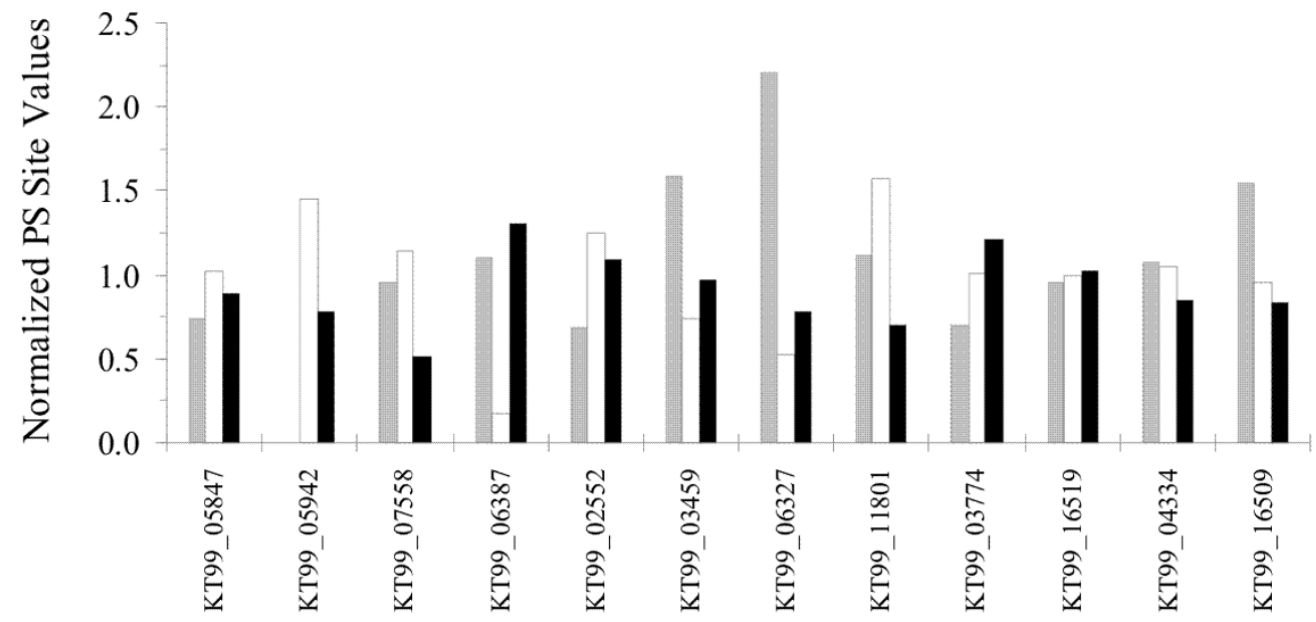

\section{Figure 4}

Distribution of PS with respect to membrane protein topology. The normalized fraction of trans-membrane PS sites (black columns) is compared to the cytoplasmic (grey) and external (white) PS sites. Results are shown only for Vibrionaceae (A) and Shewanellaceae (B) transporters. KT99 TrEMBL IDs are not available, for this reason in (B) are reported NCBI's Locus Tags.

allowed us to identify both specific and common functional categories involved in SS9 and KT99 adaptation strategies. In fact, both of them are piezophiles but they were isolated from distinct environments and showed optimum growth at different pressures and temperatures.

\section{Functional classes}

To clarify the role of PS genes we analyzed their statistical over-representations in all functional classes. As expected evaluation of both COG and GO results confirmed the key role of transport proteins in abyssal adaptation. It is known from the literature that the transport of com- 
pounds like Trp, Lys, His and Leu is reduced at high pressure, owing to the volume change of activation needed in the transport process [2]. In fact, the tat 2 gene enables transformed yeast cells to grow at high hydrostatic pressure by encoding a high-affinity tryptophan permease which counteracts the significant inhibition of tryptophan uptake into the cells due to high pressure [20]. It was previously determined that SS9 up-regulates certain transporters mainly at low pressure. This could indicate that these proteins may have evolved a particular structure adapted to elevated pressure because their up-regulation could compensate the reduction of functionality.

Five PS genes previously identified as differentially expressed in microarray experiments probably play a crucial role (Additional file 1). Among these, there are three permease components that are part of an $\mathrm{ABC}$ transporter: "hypohetical ABC transporter, permease protein" (PBPRA2115); "putative ABC-type arginine transport system, permease component" (PBPRA2740); "putative ABC-type metal ion transport system, permease component" (PBPRA2941). The remaining are a "periplasmic peptide binding protein" belonging to a putative peptide ABC transporter (PBPRA0525) and a "putative glycerol-3phosphate transporter" (PBPRA0158). PBPRA0525 is part of the ABC transporter coded by PBPRA0521-PBPRA0525 that was previously shown to be up-regulated at $28 \mathrm{MPa}$ and $4{ }^{\circ} \mathrm{C}$, while a second peptide $\mathrm{ABC}$ transporter, coded by PBPRA2934-PBPRA2938 genes, was down-regulated at $28 \mathrm{MPa}$. These data indicated that SS9 can express proteins adapted to different pressures [6]. The fact that only PBPRA0525 was identified in our study is a further indication that this protein has been selected to work better in the abyssal environment, and gives indirect evidence that SS9 probably evolved from some mesophilic strains.

Moreover, the number of PS sites in the piezophile permease compared to those of the mesophiles is twice (66) 559 versus 30/608) in PBPRA0525, which is over expressed at high pressure and low temperature.

Another class of transporters that are influenced by pressure is that of the voltage-gated channels-. Using the patch clamp technique, it has been demonstrated that pressure acts on the movement of the charge sensor and on the conformational change involved in opening the channel pore [21-23]. For certain channels the $\Delta \mathrm{V}$ value for the closed/open transition is $-105 \mathrm{ml} \mathrm{mol}^{-1}$, that is, the open state occupies a smaller volume than the closed state [24] thus implying that pressure could have a large influence on this process. In this work we verify that in deep-sea organism transporters there are PS sites, a finding that had previously been hypothesized -by other authors [21]. --In light of these points the demonstration that many PS genes belong to the GO transport category is noteworthy.
Despite the high number of different classes of transporters made difficult to discuss the role of the localization of the PS sites on protein structure, their high frequency in the external part of the membrane proteins is noteworthy.

Another significant group of proteins is that involved in protein export across the outer membrane [25]. Two of them, "protein export protein SecD" (PBPRA0745) and "protein export protein SecF" (PBPRA0746), belong to the translocation complex and were identified as positive selected (Additional file 4). SecDF of B. subtilis is implicated in early translocation steps, whereas in E. coli these polypeptides are required to release mature proteins. It was found that strains lacking intact secDF are cold-sensitive and this conditional phenotype probably reflects the thermal sensitivity of protein translocation [26]. As previously noted high pressure and low temperature have a similar effect on bacterial membranes and are probably responsible for positive selection acting on these two proteins and globally on SS9 transporters [27]. The maintenance of biological membranes in a narrow range of viscosity, homeoviscous response, or within a liquid-crystalline phase, homeophasic response, is essential for growth and survival $[28,29]$. This is not surprising, because a great number of biological processes such as membrane transport, protein-protein interactions within the lipid bilayer, metabolic electron transport, intracellular signaling and gene regulation are dependent on a suitable membrane physical structure [30]. Due to the key role played by membrane proteins, we computed the distribution of PS sites in different domains; however, our analysis showed that in $40 \%$ of the proteins, considering normalized values, the higher fraction of PS sites is located in the external part and not in trans-membrane region, the opposite of what was expected considering the putative influence of the membrane composition mentioned above. This finding is in agreement with similar results obtained in mouse, where, compared with the intracellular (cytoplasmic and nuclear) domains, a greater proportion of extracellular domains possess higher $\omega$ values [13] and are subject, on average, to greater positive diversifying selection. Moreover other studies comparing transporters of Bacteria, Archea and Eucarya [31] indicate that the major differences in terms of size between orthologs are in the hydrophilic regions, the most important from a regulatory point of view.

Some of the SS9 PS orthologs we identified belong to the GO categories related to the following terms: "Locomotion", "Ciliary or flagellar motility" and "Cell motility" (Additional file 3). This is probably related to the importance of bacterial motility in enhancing bacteria-organicmatter coupling and reducing the ability of protozoa to graze on bacteria [32]. It was shown that the P. profundum deep sea adapted strains (SS9 and DSJ4) contain an addi- 
tional gene cluster absent in the shallow bathytype (3TCK) which resembles genes for the production of lateral flagella [6]. The function of this additional cluster is unknown, but preliminary results have provided evidence that the SS9 strain displays high motility at $28 \mathrm{MPa}$ pressure and swims very slowly at atmospheric pressure, while the opposite phenotype has been observed in the shallow water 3TCK [33]. Proteins coded by the PS genes, which we recognized as part of this pathway, are the "putative flagellar basal-body rod protein FlgF" (PBPRA0905) and the "putative polar flagellar FlgG" (PBPRA0906) located on the external membrane region. Also involved in this process are the "flagellar basal body-associated protein FlgB" (PBPRA0901) and the "flagellar basal body protein FliH" (PBPRA0925), both part of the motor switch (Additional file 4). Flagellar assembly and motor function are known to be the most pressure-sensitive cellular processes $[4,34]$ therefore we hypothesized this modification of flagellar structure to be a strategy specific to SS9. In fact in the KT99 PS gene list there are no flagellar proteins and the only one related to the cell motility class is "MSHA biogenesis protein MshL" (KT99_07703), part of the Type II secretion system.

We have already stated that independent analysis done on Shewanellaceae and Vibrionaceae allowed the detection of functional classes involved in SS9 and KT99 specific and common adaptations. In order to further investigate the most interesting pathways we compared the two PS gene lists using BLAST and we found 12 genes shared by them (Table 3 ). They are respectively $18 \%$ and $5.6 \%$ of the corresponding total of PS genes. Among the proteins coded by them there are three transporters and this stresses their importance in deep-sea adaptation.

The presence of the "hypothetical Primosomal Replication Protein N" is relevant as it has a role in the assembly of the primosome and has a helicase activity [35]. The same function is present in RecD protein of SS9 that also enhances the pressure resistance in E. coli [36].

Positive selection acting on this protein could counteract the reduction in DNA and RNA biosynthesis at high pressure in mesophiles but also the inhibition of cellular division that could determine the formation of highly filamentous cells $[37,38]$.

\section{Localization of PS sites in the Protein Structure}

It has been proposed that solvent exposed amino acids have a lower purifying selection and for this reason could vary more easily. An explanation could be the lower functional constraint of the residues located on the protein surface with respect to the buried residues. For most of the proteins this is clearly evidenced from the PhyMol software generated images (Figure 2). To verify this assump- tion we computed the fraction of solvent exposed PS sites in 41 SS9 proteins for which a modeled structure was available in database. Comparing the fraction of the solvent-exposed amino acids with that of the PS sites, we found the latter higher in $93 \%$ of the proteins. Despite the higher tendency of the exposed amino acids to change, we could hypothesize that protein surface has a role in deepsea adaptation. For example [39] it has been reported that the increased exposure of hydrophobic residues and the reduction of solvent exposed charged amino acids determines a destabilization of the protein surface. This is linked to cold adaptation, reduction of activation energy and to an increased catalytic efficiency $[40,41]$.

\section{Conclusion}

In this paper we propose a new statistical approach that could be applied to identify genes under positive selection by means of omega value calculation. Using this method we investigated the problem of molecular adaptation in deep-sea adapted bacteria. Genes obtained in vibrionaceae and shewanellaceae were grouped using COG and GO and the results showed that positive selection in piezophiles targets a wide range of different functions such as transport of solutes, protein translocation, DNA synthesis and flagellar motility. Transport was identified as the most notable biological process that underwent positive selection in the two deep-sea bacteria that we studied. This result is particularly interesting because it confirms some previous findings obtained by other approaches such as the analysis of expression profiles at high and low pressure [2] and the analysis of laterally transferred genes in deep-sea bacteria [6]. We can therefore conclude that the proteins involved in transport represent a bona fide class of proteins that requires consistent modifications to adapt to the deep-sea environment.

Structural mapping of PS sites in membrane proteins reveals that they tend to occur preferentially in extracellular regions of membrane proteins.

\section{Methods}

Among the completely sequenced bacterial genomes deposited in the NCBI database [42], we chose those with the larger number of orthologous genes with respect to the two piezophiles that we wanted to investigate. To select these microorganisms all protein-coding gene sequences in SS9 and KT99 were blasted -respectively against the Vibrionaceae and Shewanellaceae available at NCBI's ftp site [43]. BLAST results were parsed using PERL scripts in order to select only those orthologs satisfying alignment criteria defined in literature [14]: alignment extension over $70 \%$ and e-value cut-off $10^{-5}$. All identity values were higher than $40 \%$ and most of e-value indicated an exact match. To discard paralogous genes from our analysis, reciprocal BLAST searches were performed 
and the selection was limited at best reciprocal hits. Then we selected the definitive orthologous genes choosing those of the three mesophilic bacteria with the higher number of matches in the pair comparison with the piezophile. Only SS9 or KT99 proteins with a valuable scoring match in each of the three mesophilic bacteria were considered. All the following procedures were carried out separately for Shewanellaceae and Vibrionaceae families.

\section{Tests for positive selection}

The positive selection analysis was performed considering all the possible pairs of bacteria and six $\omega$ values series were calculated. This kind of test required a specific DNA alignment for each gene based on the corresponding protein alignment. For this reason, orthologous protein sequences were aligned with ClustalW [44] and then were back-translated to the corresponding DNA sequences using RevTrans software. This allowed the preservation of gaps obtained during protein alignment, essential to avoid in-dels causing frameshifts in DNA sequences.

To test for positive selection each ortholog pair of the six series was compared using the method implemented in yn00 software of PAML package [45] that evaluates the presence of PS sites calculating the $\mathrm{dN} / \mathrm{dS}$ ratio $(\omega)$ between sequences pairs. The results were six groups of values: three from the comparison of the piezophilic bacteria with the mesophiles and three from the mesophilic bacteria between them.

\section{Codon Adaptation Index (CAI)}

CAI values for orthologous genes were obtained for all bacteria using CodonW [46]. This software performs correspondence analysis of the Relative Adaptiveness (RA) of the codon usage of a gene towards the codon usage of highly expressed genes. RA of each codon is the usage ratio with respect to that of the most abundant codon for the same amino acid. This estimate was done selecting genes identified in SS9 and S. oneidensis MR-1 from those having high expression values (fluorescence arbitrary units higher than 10000) in microarray experiments performed on these bacteria. SS9 microarray results were obtained from ArrayExpress database (E-MEXP-210) [47], instead S. oneidensis's ones where taken from GEO database (GSM100358, GSM100396, GSM100872, GSM100873，GSM87832，GSM88468， GSM88473， GSM88474) [48].

\section{SAM analysis}

The six values reckoned for each ortholog were therefore analyzed in order to identify those significantly higher in the comparison between piezophilic and mesophilic bacteria with respect to the values obtained from the mesophiles only. These genes could possibly be affected by positive selection in piezophiles (PS genes) and could play a role in bacterial adaptation to deep-sea environment.

Statistical analysis was performed using SAM [49]. This software was initially developed for microarray data analysis and implements a modified t-test useful to verify large number of independent hypothesis. SAM employs repeated permutations to settle which of the values obtained in the piezophile-mesophiles comparisons are significantly higher than those identified comparing mesophiles only. The cutoff for significance is determined by a tuning parameter delta, chosen by the user and based on the false discovery rate (FDR) set at $10 \%$. All the following bioinformatics analyses were executed on PS genes lists obtained as described above.

\section{COG and GO functional classes}

PS genes were evaluated to identify enrichment of GO and COG categories relative of what would have been expected by chance alone.

While GO enrichment was calculated using GoMiner software [50], the COG calculation was done according to the hypergeometric distribution. With this analysis we identified the chance of observing the number of genes annotated with a particular COG category among the selected group of orthologs. The probability P of finding at least $k$ genes of a functional category within a group of $n$ genes is given by:

$$
P=\sum_{i=k}^{n} \frac{\left(\begin{array}{c}
f \\
i
\end{array}\right)\left(\begin{array}{c}
g-f \\
n-i
\end{array}\right)}{\left(\begin{array}{l}
g \\
n
\end{array}\right)}
$$

where $f$ is the total number of genes within the same category (in the matrix) and $g$ is the total number of genes identified using SAM software. Hypergeometric distribution was calculated using R statistical package [51] and the significance threshold considered is 0.05 .

\section{Fast evolving regions prediction}

A variant of the TL method [52] was used on the PS genes to detect regions with significantly higher variability within each sequence pairs. The number of nonsynonymous mutations is estimated on every codon position in the alignment and regions with overabundant mutation rate are reckoned using Faster software [18]. These sections were further selected and considered in the study only if present in piezophile-mesophiles comparisons and discarded if also identified comparing mesophiles. 


\section{PS sites mapping on 3D structures of proteins in Vibrionaceae}

When available, we downloaded from ModBase the 3D structure PDB file calculated by comparative modeling for each PS gene [53]. To get more reliable results we considered only the proteins with a percentage of identical residues higher than $40 \%$ in the alignment between the target and the template. For each ortholog we aligned the three mesophile sequences first independently and then with the piezophile sequence using ClustalW. In this way we identified amino acids conserved among the three but variable with respect to the piezophile sequence. We considered as variable only those amino acids having strongly different physical-chemical properties. These sites, along with the position of the ligand binding site downloaded from ModBase, were highlighted in the 3D protein structure using PyMol software. We also mapped in the structure the position of the overabundant mutation region, when present. Then the DSSP software was utilized to estimate solvent accessible surface areas (ACCs) for all residues of each ortholog PDB file selected above [54]. Finally we compared each protein mean ACC value with those obtained only for the PS sites.

\section{PS sites localization on trans-membrane proteins}

For each selected membrane protein we estimated the trans-membrane helices positions and the protein orientation, as predicted by Phobius software [55]. Therefore we localized the PS sites position in all sequences and we mapped them with respect to the protein topology, inside or outside the membrane, and on trans-membrane helices. For each of these sections the number of PS sites was normalized for their total number and the protein region length. The values for exposed (ext), trans-membrane (tr) and cytoplasmic (cyt) PS sites are given by:

(ext $\mathrm{N}^{\circ}$ of PS sites $/$ tot $\mathrm{N}^{\circ}$ of PS sites $) /\left(\right.$ ext $\mathrm{N}^{\circ}$ of AA/tot $\mathrm{N}^{\circ}$ of $\mathrm{AA})$

(cyt $\mathrm{N}^{\circ}$ of PS sites/tot $\mathrm{N}^{\circ}$ of PS sites)/(cyt $\mathrm{N}^{\circ}$ of $\mathrm{AA} /$ tot $\mathrm{N}^{\circ}$ of $\mathrm{AA}$ )

(tr $\mathrm{N}^{\circ}$ of PS sites $/$ tot $\mathrm{N}^{\circ}$ of PS sites) $/\left(\operatorname{tr} \mathrm{N}^{\circ}\right.$ of $\mathrm{AA} / \operatorname{tot} \mathrm{N}^{\circ}$ of $\mathrm{AA})$.

\section{Authors' contributions}

SC conceived of the study, performed the statistical and Gene Ontology analysis, participated in the interpretation of the results and drafted the manuscript.

LT participated in the design of the study, developed the PERL scripts, calculated the omega values, participated in the interpretation of the results and drafted the manuscript.
GV participated in the design and in the coordination of the study and revised the manuscript. All authors read and approved the final manuscript.

\section{Additional material}

\section{Additional file 1}

Additional information on PS genes identified in Vibrionaceae family. In columns A-H are reported respectively: internal database ID, NCBI Locus Tag, TrEMBL ID, NCBI gene annotation, COG category, statistic q-value, total number of amino acids and of PS sites for each P. profundum SS9 orthologue. The $\omega$ values obtained with PAML software for the six pair-comparisons and CAI values calculated for each bacterium are reported in I-R; percentage of total solvent-exposed amino acids and solvent-exposed PS sites are shown in columns $S$ and $T$. In the table are also highlighted differentially expressed genes identified in SS9 microarray experiments (yellow background), common PS genes between SS9 and KT99 (underlined), piezosensitive mutants (italic), genes with overabundant mutation region (red), and proteins with modeled $3 D$ structure (bold). Organism names were abbreviated as follows: P. profundum SS9 $(P)$, V. vulnificus (V1), V. parahaemoliticus (V2) and V. fisheri (V3). Click here for file

[http://www.biomedcentral.com/content/supplementary/14712148-8-313-S1.xls]

\section{Additional file 2}

Additional information on PS genes identified in Shewanellaceae family. In colums A-H are reported respectively: internal database ID, NCBI Locus Tag, TrEMBL ID, NCBI gene annotation, COG category, statistic q-value, total number of amino acids and of PS sites for each $S$. benthica KT99 orthologue. All reported TrEMBL IDs and COG annotations refer to $\mathrm{S}$. oneidensis MR1. The $\omega$ values obtained with PAML software for the six pair-comparisons and CAI values calculated for each bacterium are reported in $M$ and $N$. In the table are also highlighted common PS genes between SS9 and KT99 (underlined) and genes with overabundant mutation regions (red). Organism names are abbreviated as follows: S benthica KT99 (P), S. oneidensis (S1), S. frigidimarina (S2) and S. baltica (S3).

Click here for file

[http://www.biomedcentral.com/content/supplementary/14712148-8-313-S2.xls]

\section{Additional file 3}

GO categories enriched with PS genes in Shewanellaceae and Vibrionaceae* $^{*}$. Columns represent respectively: functional classes, GO codes, number of PS genes for each category, total number of orthologous genes for each category and p-value calculated using GoMiner software for Shewanellaceae (columns 3, 4, 5) and Vibrionaceae (columns 6, 7, 8). In bold are highlighted the significant values and the gray background indicates the enriched classes both in Shevanellaceae and Vibrionaceae. The asterisk indicates that the functional class names have been shortened, please refer to the GO code to recover the correct annotation term. Click here for file

[http://www.biomedcentral.com/content/supplementary/14712148-8-313-S3.xls] 


\section{Additional file 4}

KEGG schematic representation of Flagellar assembly (A) and Protein export Sec pathway (B). PS genes of SS9 were highlighted in blue on KEGG pathways and modules. As discussed in the text both motility and transport contain a high number of PS genes.

Click here for file

[http://www.biomedcentral.com/content/supplementary/14712148-8-313-S4.doc]

\section{Acknowledgements}

We thank Chiara Romualdi for help and suggestions in statistical analysis and Georgine Faulkner for critical reading of the manuscript.

\section{References}

I. Yayanos AA: Microbiology to $\mathbf{1 0 , 5 0 0 ~} \mathrm{m}$ in the deep sea. Annu Rev Microbiol 1995, 49:777-805.

2. Vezzi A, Campanaro S, D'Angelo M, Simonato F, Vitulo N, Lauro FM, Cestaro A, Malacrida G, Simionati B, Cannata N, Romualdi C, Bartlett DH, Valle G: Life at depth: Photobacterium profundum genome sequence and expression analysis. Science 2005, 307:| 459-| |46 I.

3. DeLong EF, Franks DG, Yayanos AA: Evolutionary relationships of cultivated psychrophilic and barophilic deep-sea bacteria. Appl Environ Microbiol 1997, 63:2105-2108.

4. Bartlett $\mathrm{DH}$ : Pressure effects on in vivo microbial processes. Biochim Biophys Acta 2002, 1595:367-381.

5. Kato C, Nogi Y: Correlation between phylogenetic structure and function: examples from deep-sea Shewanella. FEMS Microbiol Ecol 2000, 35:223-230.

6. Campanaro S, Vezzi A, Vitulo N, Lauro FM, D'Angelo M, Simonato F, Cestaro A, Malacrida G, Bertoloni G, Valle G, Bartlett DH: Laterally transferred elements and high pressure adaptation in Photobacterium profundum strains. BMC Genomics 2005, 6:122.

7. Nogi Y, Masui N, Kato C: Photobacterium profundum sp. nov., a new, moderately barophilic bacterial species isolated from a deep-sea sediment. Extremophiles 1998, 2:1-7.

8. Swarz JR, Colwell RR: Effect of hydrostatic pressure on growth and viability of Vibrio parahaemolyticus. Appl Microbiol 1974, 28:977-98।.

9. Nei M, Gojobori T: Simple methods for estimating the number of synonymous and nonsynonymous nucleotide substitutions. Mol Biol Evol 1986, 3:418-426.

10. Yang Z, Nielsen R: Estimating synonymous and nonsynonymous substitution rates under realistic evolutionary models. Mol Biol Evol 2000, 17:32-43.

II. Rocha EPC, Danchin A: An analysis of determinants of amino acids substitution rates in bacterial proteins. Mol Biol Evol 2004, 21: 108-116.

12. Simonato F, Campanaro S, Lauro FM, Vezzi A, D'Angelo M, Vitulo N, Valle G, Bartlett DH: Piezophilic adaptation: a genomic point of view. J Biotechnol 2006, I 26: I I-25.

13. Mouse Genome Sequencing Consortium: Initial sequencing and comparative analysis of the mouse genome. Nature 2002, 420:520-562.

14. Tatusov RL, Koonin EV, Lipman DJ: A genomic perspective on protein families. Science 1997, 278:631-637.

15. Ashburner M, Ball CA, Blake JA, Botstein D, Butler H, Cherry JM, Davis AP, Dolinski K, Dwight SS, Eppig JT, Harris MA, Hill DP, IsselTarver L, Kasarskis A, Lewis S, Matese JC, Richardson JE, Ringwald M, Rubin GM, Sherlock G: Gene Ontology: tool for the unification of biology. The Gene Ontology Consortium. Nat Genet 2000, 25:25-29.

16. Kanehisa M, Goto S: KEGG: Kyoto Encyclopedia of Genes and Genomes. Nucl Acid Res 2000, 28:27-30.

17. MOD BASE: Database of Comparative Protein Structure Models [http://modbase.compbio.ucsf.edu/modbase-cgi/index.cgi]

18. Zheng $Y$, Roberts RJ, Kasif S: Identification of genes with fastevolving regions in microbial genomes. Nucl Acid Res 2004 32:6347-6357.
19. Lauro FM, Tran K, Vezzi A, Vitulo N, Valle G, Bartlett DH: Largescale transposon mutagenesis of Photobacterium profundum SS9 reveals new genetic loci important for growth at low temperature and high pressure. J Bacteriol 2008, 190:1699-1709.

20. Abe F, Horikoshi K: Tryptophan permease gene TAT2 confers high-pressure growth in Saccharomyces cerevisiae. Mol Cell Biol 2000, 20:8093-8102.

21. Macdonald AG: Ion channels under high pressure. Comp Biochem Physiol A Mol Integr Physiol 2002, I 3 I:587-593.

22. Conti F, Inoue I, Kukita F, Stühmer W: Pressure dependence of sodium gating currents in the squid giant axon. Eur Biophys J 1984, II:137-147.

23. Kukita F: Solvent effects on squid sodium channels are attributable to movements of a flexible protein structure in gating currents and to hydration in a pore. J Physiol 2000, 3:357-373.

24. Macdonald AG: Effect of high hydrostatic pressure on the BK channel in bovine chromaffin cells. Biophys J 1997, 73:1866-1873.

25. Robson A, Collinson I: The structure of the Sec complex and the problem of protein translocation. EMBO Rep 2006, 7:1099-1103.

26. Bolhuis A, Broekhuizen CP, Sorokin A, Van Roosmalen ML, Venema G, Bron S, Quax WJ, van Dijl JM: SecDF of Bacillus subtilis, a molecular siamese twin required for the efficient secretion of proteins. J Biol Chem 1998, 273:21217-21224.

27. Allen EE, Bartlett DH: Structure and regulation of the omega-3 polyunsaturated fatty acid synthase genes from the deep-sea bacterium Photobacterium profundum strain SS9. Microbiology 2002, I 48:1903-1913.

28. Sinensky M: Homeoviscous adaptation - a homeostatic process that regulates the viscosity of membrane lipids in Escherichia coli. Proc Natl Acad Sci 1974, 71:522-525.

29. McElhaney RN: Effects of membrane lipids on transport and enzymic activities. In Current topics in membranes and transport Edited by: Razin S, Rottem S. New York: Academic Press; 1982:317-380.

30. Kadner RJ: Cytoplasmic membrane. In Escherichia coli and Salmonella: cellular and molecular biology 2 nd edition. Edited by: Neidhardt FC, Curtiss R III, Ingraham JL, Lin ECC, Low KB, Magasanik B, Reznikoff WS, Riley M, Schaechter M, Umbarger HE. Washington DC: ASM Press; 1996:58-87.

31. Chung YJ, Krueger C, Metzgar D, Saier MH Jr: Size comparisons among integral membrane transport protein homologues in bacteria, Archaea, and Eucarya. J Bacteriol 2001, 183:1012-1021.

32. Grossart HP, Riemann L, Azam F: Bacterial motility in the sea and its ecological implications. Aquat Microb Ecol 200I, 25:247-258

33. Lauro FM, Bartlett DH: Prokaryotic lifestyles in deep sea habitats. Extremophiles 2008, I 2:15-25.

34. Meganathan R, Marquis RE: Loss of bacterial motility under pressure. Nature 1973, 246:525-527.

35. Zavitz KH, DiGate RJ, Marians KJ: The priB and priC replication proteins of Escherichia coli. Genes, DNA sequence, overexpression, and purification. J Biol Chem I99/, 266:। 3988- 13995.

36. Bidle KA, Bartlett $D H$ : RecD function is required for high-pressure growth of a deep-sea bacterium. I Bacteriol 1999, 181:2330-2337.

37. ZoBell $C E$, Cobet $A B$ : Growth, reproduction, and death rates of Escherichia coli at increased hydrostatic pressures. J Bacteriol 1962, 84: 1228-1236.

38. Yayanos AA, Pollard EC: A study of the effects of hydrostatic pressure on macromolecular synthesis in Escherichia coli. Biophysics 1969, 9:1464-1482.

39. Saunders NF, Thomas T, Curmi PM, Mattick JS, Kuczek E, Slade R, Davis J, Franzmann PD, Boone D, Rusterholtz K, Feldman R, Gates C, Bench S, Sowers K, Kadner K, Aerts A, Dehal P, Detter C, Glavina T, Lucas S, Richardson P, Larimer F, Hauser L, Land M, Cavicchioli R: Mechanisms of thermal adaptation revealed from the genomes of the Antarctic Archaea Methanogenium frigidum and Methanococcoides burtonii. Genome Res 2003, 13:1580-1588.

40. Zecchinon L, Claverie P, Collins T, D'Amico S, Delille D, Feller G, Georlette D, Gratia E, Hoyoux A, Meuwis MA, Sonan G, Gerday C: Did psychrophilic enzymes really win the challenge? Extremophiles 200I, 5:313-32I.

4I. Siddiqui KS, Cavicchioli R, Thomas T: Thermodynamic activation properties of elongation factor 2 (EF-2) proteins from psy- 
chrotolerant and thermophilic Archaea. Extremophiles 2002, 6:143-150.

42. ENTREZ Genome Project [http://www.ncbi.nlm.nih.gov/ genomes/lltp.cgi]

43. [ftp://ftp.ncbi.nih.gov/genomes/Bacteria/].

44. Thompson JD, Higgins DG, Gibson TJ: CLUSTAL W: improving the sensitivity of progressive multiple sequence alignment through sequence weighting, positions-specific gap penalties and weight matrix choice. Nucl Acid Res 1994, 22:4673-4680.

45. Yang Z: PAML: a program package for phylogenetic analysis by maximum likelihood. Comp Appl Biosc 1997, I 3:555-556.

46. Peden JF: Analysis of codon usage. In PhD Thesis University of Nottingham; 1999.

47. ArrayExpress database [http://www.ebi.ac.uk/arrayexpress/]

48. GEO, Gene Expression Omnibus, database [http:// www.ncbi.nlm.nih.gov/geo/]

49. Tusher VG, Tibshirani R, Chu G: Significance analysis of microarrays applied to the ionizing radiation response. Proc Natl Acad Sci 200I, 98:5II6-5I2I.

50. Zeeberg BR, Feng W, Wang G, Wang MD, Fojo AT, Sunshine M, Narasimhan S, Kane DW, Reinhold WC, Lababidi S, Bussey KJ, Riss J, Barrett JC, Weinstein JN: GoMiner: a resource for biological interpretation of genomic and proteomic data. Genome Biol 2003, 4:R28.

5I. R Development Core Team: R: A language and environment for statistical computing. Vienna, Austria, R Foundation for Statistical Computing; 2006.

52. Tang $\mathrm{H}$, Lewontin RC: Locating regions of differential variability in DNA and protein sequences. Genetics 1999, 153:485-495.

53. Sánchez R, Pieper U, Mirkoviæ N, de Bakker PI, Wittenstein E, Sali A: MODBASE, a database of annotated comparative protein structure models. Nuc Acids Res 2000, 28:250-253.

54. Kabsch W, Sander C: How good are predictions of protein secondary structure? FEBS Lett 1983, I55:179-182.

55. Käll L, Krogh A, Sonnhammer EL: Advantages of combined transmembrane topology and signal peptide prediction - the Phobius web server. Nucl Acid Res 2007, 35:W429-432.

Publish with Bio Med Central and every scientist can read your work free of charge

"BioMed Central will be the most significant development for disseminating the results of biomedical research in our lifetime. "

Sir Paul Nurse, Cancer Research UK

Your research papers will be:

- available free of charge to the entire biomedical community

- peer reviewed and published immediately upon acceptance

- cited in PubMed and archived on PubMed Central

- yours - you keep the copyright
BioMedcentral 